\title{
Sleep-Related Attentional Bias in Insomnia: Time to Examine Moderating Factors?
}

\author{
Umair Akram ${ }^{1,2 *}$, Nicola L. Barclay ${ }^{2}$ and Bronwyn Milkins ${ }^{3}$ \\ ${ }^{1}$ Department of Psychology, Sociology and Politics, Sheffield Hallam University, Sheffield, United Kingdom, ${ }^{2}$ Nuffield \\ Department of Clinical Neurosciences, Sir William Dunn School of Pathology, Sleep and Circadian Neuroscience Institute, \\ University of Oxford, Oxford, United Kingdom, ${ }^{3}$ Elizabeth Rutherford Memorial Centre for the Advancement of Research on \\ Emotion, University of Western Australia, Perth, WA, Australia
}

Keywords: insomnia, attentional bias, 5HTTLPR polymorphism, worry, misperception

\section{OPEN ACCESS}

Edited by:

Nicola Cellini,

Università degli Studi di Padova, Italy

Reviewed by:

Markus Jansson-Fröjmark, Karolinska Institutet (KI), Sweden

Heather Cleland Woods,

University of Glasgow,

United Kingdom

${ }^{*}$ Correspondence:

Umair Akram

u.akram@shu.ac.uk

Specialty section:

This article was submitted to

Cognition,

a section of the journal

Frontiers in Psychology

Received: 28 August 2018 Accepted: 30 November 2018 Published: 14 December 2018

Citation:

Akram U, Barclay NL and Milkins B (2018) Sleep-Related Attentional Bias in Insomnia: Time to Examine Moderating Factors?

Front. Psychol. 9:2573.

doi: 10.3389/fpsyg.2018.02573
Prominent cognitive models of insomnia have emphasized the notion that the disorder is in part maintained by an attentional bias for sleep related "threat" cues which may be internal (i.e., bodily sensations) or external (i.e., environmental) in nature (Harvey, 2002; Espie et al., 2006). To support this proposition, a growing number of studies have examined the presence of a sleep-related attentional bias for words and images using experimental tasks including the dot-probe, flicker, Posner, emotional Stroop, and eye-tracking paradigms (see Harris et al., 2015 for a review). Many of these studies have provided encouraging evidence for the presence of such a bias in insomnia. However, the evidence base advocating the presence of such a bias remains mixed with a number of studies yielding no statistically significant effects. While a recent review (Harris et al., 2015) cautiously suggests biased attention for sleep-related threat information to be a likely feature of insomnia, the authors highlight the need to understand the specificity of this bias and its relationship with mechanisms believed to underpin the disorder (e.g., sleep preoccupation). Furthermore, whilst it is possible that the mixed evidence may stem from methodological differences relating to the task or population used, the possible moderating influence of these factors on the relationship between attentional bias for sleep-related threat information and insomnia have only recently been examined (e.g., Zheng et al., 2018). With this in mind, we propose candidate factors that may play a crucial role in addressing moderating questions such as "when," "for whom" and "under which" conditions are sleep-related attentional biases evident in individuals characterized by insomnia.

\section{HTTLPR POLYMORPHISM AND BRAIN REACTIVITY}

First, we consider the role of the serotonin transporter polymorphism (5HTTLPR). Some authors have demonstrated the 5HTTLPR short allele to be related to an increased risk for insomnia/poor sleep quality relative to the long allele (Deuschle et al., 2010; Huang et al., 2014), though findings are inconsistent (e.g., Barclay et al., 2011). Others have documented that the association between 5HTTLPR polymorphism and risk for insomnia/poor sleep is moderated by life stress (Brummett et al., 2007; Huang et al., 2014); and recently the short allele has been associated with increased hypothalamic-pituitary-adrenal (HPA) axis reactivity in response to stress (van Dalfsen and Markus, 2015). However, this association is moderated by sleep quality: long-long homozygotes experiencing poor sleep quality exhibit heightened stress reactivity relative to shortshort homozygotes (van Dalfsen and Markus, 2015). Regardless of the mechanisms involved, it appears that 5HTTLPR genotype, sleep quality and stress reactivity are intricately linked, and one possibility is that 5HTTLPR genotype may differentially alter an individual's reactivity to stress under different environmental conditions. In the context of attention bias, it is possible that 
5HTTLPR genotype differentially affects behavioral outputs, dependent on sleep quality. In other words, it is possible that 5HTTLPR polymorphisms differentially affect biased attention for "threat" relevant stimuli (possibly due to differences in the activity of the HPA axis) in good vs. poor sleepers.

In other psychiatric populations (e.g. anxiety/depression), a relationship between the short allele and an attentional bias for emotionally salient words and images has been evidenced (Beevers et al., 2009; Fox et al., 2009; Perez-Edgar et al., 2010). Specifically, this allelic variation has been linked with difficulty disengaging attention away from response to threat (Beevers et al., 2009), whereas the long allele has been associated with threat avoidance and increased attention for positively valanced stimuli (Hariri et al., 2002; Munafò et al., 2008). It would be worthy to determine whether a similar pattern would occur in insomnia patients, or whether the presence of poor sleep in this population reverses the 5HTTLPR association as would be expected in light of van Dalfsen and Markus's (2015) finding. Either way, we hypothesize that allelic variation in the 5HTTLPR polymorphism to also modulate the relationship between insomnia and attentional bias.

Given that the amygdala is involved in the processing of emotional information, and because one of the roles of serotonin is regulating mood (Ressler and Nemeroff, 2000), researchers have examined whether 5HTTLPR genotype differentially affects neural responses of the amygdala to emotional stimuli. In healthy participants, short allele carriers exhibit greater neural activity in the amygdala, and greater connectivity between the amygdala and ventromedial prefrontal cortex, compared to longlong homozygotes when presented with negatively toned stimuli (Pezawas et al., 2005; Heinz et al., 2007). Given that individuals with insomnia display increased amygdala reactivity in response to sleep-related stimuli (which may be interpreted as negative; Baglioni et al., 2014) it is plausible that 5HTTLPR genotype may underlie this neural response, with genetically vulnerable individuals exhibiting greater sensitivity, and possibly greater attention, to potential threats.

\section{VALANCE OF MOOD STATE}

Second, previous research has statistically controlled for traitlike emotional distress characterized by disposition to experience anxiety or depression (Jansson-Fröjmark et al., 2012; Akram et al., 2018c). However, attentional bias to threat is a transient process (MacLeod et al., 2002) and emotional tone has differential effects. For example, dysphoric mood is often associated with negative cognitive activity (e.g., catastrophising, worry, rumination) whereas this can be attenuated with enhanced mood (Baglioni et al., 2010, 2014). As such, current mood state should be taken into account in future studies rather than just trait-like characteristics. Indeed, inducing a negative mood state in people with insomnia influences the extent to which an attentional bias to threatening information is displayed (Zheng et al., 2018). Specifically, when assigned to a negative mood inducing (i.e., autobiographical recall of poor sleep) or control (i.e., reading recall) condition prior to completing a dot-probe task comprised of images differing in emotional valence and relatedness to sleep (i.e., general threat, sleep positive, sleep negative), an overall attentional bias to all images emerged only amongst individuals with insomnia who were subject to negative mood state induction Despite an absence of evidence for a sleep-related attentional bias in this study, it remains possible that the overall valance of an individual's emotional mood state, as well as its intensity, duration and frequency of occurrence, may act as moderating factors. That said, further research is required to clarify this position, and expand on these novel findings by examining the therapeutic potential of a positive mood state induction.

\section{SLEEP-RELATED WORRY}

Next, we speculate that attentional bias for threatening information might extend particularly to individuals who attribute worry as the main cause of their insomnia. Worry, by definition, is a cognitive process that involves a chain of emotionally negative thoughts about potential future events (Hirsch and Mathews, 2012). Whilst worry may be utilized in a productive manner in terms of solving a specific problem, it appears to be unproductive in the context of sleep, as evidenced by the consistent relationship between worry about sleep and longer sleep onset latency (Harvey, 2002; Harvey and Greenall, 2003; Weise et al., 2013). Indeed, research has consistently evidenced strong relationships between a tendency to worry and high levels of insomnia symptoms (Watts et al., 1994; Jansson and Linton, 2006; Carney et al., 2010; O'Kearney and Pech, 2014). In addition, this population displays a greater propensity to worry during the pre-sleep period, than good sleepers (e.g., Lancee et al., 2017a). Given that by nature, worry is a negative affect-laden process, it is plausible that individuals with insomnia exhibit greater attentional selectivity of emotionally negative information. Therefore, a factor that may moderate this relationship is the degree to which those with insomnia differ in experiencing increased sleep-related worry prior to initiating sleep (e.g., the consequences of not getting enough sleep on functioning; their perceived lack of ability to fix their sleep problem) which is inherently unproductive.

Potentially, a population of those with insomnia who excessively worry about the immediate and longer-term consequences of sleeplessness eventually develop an attentional bias to sleep-related negative information due to an increased personal relevance of this type of information (Espie, 2002). In contrast, those with insomnia who do not worry specifically about sleep are less likely to develop an attention bias to sleeprelated negative information. If true, empirical studies would observe a sleep-related attentional bias only in individuals for whom sleep is a major concern on nights where this more profoundly manifested (e.g., attention to signs of wakefulness such as racing heart beat and racing thoughts). While prior research has examined whether cognitive activity in general and worry differentially predict insomnia (Wicklow and Espie, 2000; Harvey and Greenall, 2003), to the best of the author's knowledge no study thus far has examined the specific possibility that differences in the content and intensity of worries (i.e., 
worry about sleep vs. worry about other topics) differentially predict attentional bias in insomnia. However, prior research has obtained suggestive evidence consistent with this latter possibility in the context of catastrophic worry and sleep-related threat (Barclay and Gregory, 2010; Barclay and Ellis, 2013). In particular, Barclay and Gregory (2010) asked poor and good sleepers to: catastrophise (i.e., iterate negative aspects of a problem: Kendall and Ingram, 1987) about their sleep and a personal worry; and to iterate a hypothetical topic in a positive manner. Here, whilst poor sleepers catastrophised more for each topic compared to good sleepers, the frequency of catastrophic worry didn't vary by topic (i.e., sleep-related worry, personal worry, or hypothetical) for poor sleepers. Additionally, these outcomes were mediated by anxiety. The authors suggest that poor sleepers' orientation of catastrophic thoughts may not always be sleep-specific; rather related to a perseverative iterative style, fuelled by anxiety. Next, Barclay and Ellis (2013) observed that poor sleepers were slower to detect sleep-related stimuli compared to non-sleep-related negative stimuli, and this pattern was not observed in good sleepers. The authors speculate that the personal relevance of the "threat" differentially impacts speed of response: sleep-related stimuli hindered performance, whereas conversely non-specific threats facilitated performance. Taken together, these findings suggest that cognitive styles (i.e., worry focused) and personal relevance of the sleep-related stimuli may moderate the attentional bias effect and may account for the inconsistencies in the literature.

Finally, it is relevant to note that worry and rumination come under the broader cognitive process of "repetitive negative thinking" or "recurrent negative thinking" which is now recognized to be a transdiagnostic cognitive process for anxiety and depressive disorders (Gustavson et al., 2018). First, however we propose examining differential aspects of thought and how this is presented in relation to insomnia and then subsequently determining whether these specific insomnia-relevant constructs moderate the relationship between attentional bias and insomnia. In turn, this area could learn whether the "repetitive negative thinking" phenomenon in anxiety and depressive disorders can be extrapolated to insomnia and other sleep disturbances.

\section{MISPERCEPTION OF SLEEP AND DAYTIME IMPAIRMENT}

It is possible that individuals with insomnia exhibiting a sleep-related attentional bias may be experiencing increased misperception pertaining to their nocturnal sleep and the extent of their daytime impairment resulting from poor sleep. Specifically, it is theorized that increased attention toward sleep-related cues during sleep and the pre-sleep period may distort the distinction between sleep and wakefulness resulting in a misperception of sleep (Harvey, 2002). Indeed, it is wellevidenced that some individuals with insomnia often misperceive attributes of sleep: self-reported sleep onset latency is usually overestimated, and total sleep time underestimated, relative to objectively recorded data (e.g., Wicklow and Espie, 2000; Tang and Harvey, 2006; Van Den Berg et al., 2008). This misperception may also extend to daytime impairment and perception of sleep-deficit. Indeed, whilst individuals with insomnia report their perception of daytime cognitive functioning (i.e., attention, working and episodic memory, problem solving) to be in a manner that confirms the presence of a sleep deficit (i.e., an increased daytime impairment: Fortier-Brochu et al., 2012), objective performance on neuropsychological measures of such functioning do not always coincide with their perception (Orff et al., 2007; Goldman-Mellor et al., 2015). In a similar manner, it has also been evidenced that individuals with insomnia consider their facial appearance to appear more physically tired than they actually are (Akram et al., 2016). However, not all those with insomnia misperceive attributes of their sleep and/or the true extent to which daytime functioning is impaired due to poor sleep. This suggests these two forms of sleep misperception (about sleep and daytime consequences) may be functionally underpinned by a third factor. One plausible factor the present commentary has alluded to is worry. It is possible that worry moderates the relationship between sleep misperception and objective measures of sleep, such that increased levels of worry serve to perpetuate misperception of sleep and of the extent to which poor sleep impairs daily functioning. In contrast, a reduced level of worry may result in more accurate perceptions of one's sleep compared with objective measures. Furthermore, worry and misperception of sleep may facilitate attention toward cues pertaining to nocturnal sleep and daytime performance as a counterproductive form of self-assessment. Here, once attention is placed on a particular cue (e.g., heart rate), it may then be interpreted in a way that confirms the sleep disturbance (e.g., "why is my heart still racing" during sleep onset) consequently feeding back to accentuate sleep-related worry (e.g., "If it doesn't slow down soon I won't be able to sleep") and misperception of sleep attributes (e.g., "I can't function at work because I slept poorly last night") in a cyclical nature (Harvey, 2002).

\section{SYMPTOM VARIATION}

Several studies investigating the presence of an attention bias in insomnia have found group differences (e.g., insomnia vs. normal-sleepers) in relation to attentional bias outcomes (Spiegelhalder et al., 2008; Jansson-Fröjmark et al., 2012; Barclay and Ellis, 2013; Beattie et al., 2017; Akram et al., 2018a,b,c; Koranyi et al., 2018). However, increased insomnia symptom severity or severity of poor sleep quality do not appear to be related to attentional bias outcomes (Spiegelhalder et al., 2008; Jansson-Fröjmark et al., 2012; Barclay and Ellis, 2013; Beattie et al., 2017; Akram et al., 2018a,b,c; Koranyi et al., 2018). With that in mind, little variation in yielded effect sizes relating to sleep-related attentional bias in insomnia are reported between clinically diagnosed patients, opportunistic samples of individuals meeting diagnostic criteria, and poor sleepers (Harris et al., 2015). That said, Spiegelhalder et al. (2010) demonstrated positive relationships between attentional bias indices and polysomnographically determined total sleep time, sleep efficiency and duration of slow-wave sleep amongst individuals with insomnia when using the dot-probe task. This pattern of findings did not extend to attentional bias when using the Stroop task. Thus, it is possible that objective measures of severity of sleep disturbance may be predictive of attention 
bias. Future research should examine the potential role of both subjective and objective variation in sleep continuity as a moderating factor of attention bias.

\section{TASK AND STIMULI}

There is mixed evidence concerning the presence of a sleeprelated attentional bias in insomnia and these inconsistencies may stem from variation in the methodological approach used. Indeed, when examining group differences (insomnia/poorsleeper vs. control) in reaction time tasks, Harris et al. (2015) determined the flicker, dot-probe and Posner tasks to demonstrate moderate to large effects sizes. In contrast, the Stroop task appears less sensitive, with two studies (Spiegelhalder et al., 2008; Barclay and Ellis, 2013) out of five (Lundh et al., 1997; Spiegelhalder et al., 2008, 2010; Zhou et al., 2018) conducted to date demonstrating an attentional bias in insomnia.

Moving forward from reaction time assessments of attentional bias, which can be considered an indirect measure of attention, a number of recent studies have employed eye-tracking paradigms with the aim to examine selective attention in insomnia (Woods et al., 2013; Beattie et al., 2017; Akram et al., 2018c). Here, visual attention can be continuously recorded throughout stimuli presentation, providing a more ecological observation of visual and selective attention relative to reaction time measures (Armstrong and Olatunji, 2012; Marks et al., 2014). Interestingly, using this methodology, only studies using sleep-related and neutral images, rather than words, as part of a free viewing task evidenced increased attention allocated to the spatial location of insomnia salient stimuli (Beattie et al., 2017; Akram et al., 2018c).

A final consideration is whether people with insomnia compared to normal sleepers differ in the extent to which they consider sleep-related stimuli as threatening. Moreover, it remains unclear whether this threat drives attentional biases in insomnia; or whether perceptions of threat are stimulated by monitoring of the external environment for sleep-related cues. This latter explanation would be consistent with the idea that the attentional bias in insomnia represents a craving for sleep rather than interpreting sleep-related stimuli as threatening.

\section{APPLICATION AND SUMMARY}

Recent evidence from the anxiety literature shows attentional bias modification (ABM) to be effective in ameliorating disorder consistent symptoms amongst individuals who elicit an attentional bias (MacLeod and Grafton, 2016). In poor sleepers, ABM administered immediately prior to bed improved subjective sleep quality and reduced pre-sleep arousal and sleep onset latency across a single sleep episode, relative to alternative nights where a control task was completed (Milkins et al., 2016). Expanding on this research, Lancee et al. (2017b) evidenced no therapeutic effect of ABM amongst those meeting diagnostic criteria for insomnia. As such, the applicability of $\mathrm{ABM}$ to insomnia remains elusive. Therefore, studies assessing the efficacy of ABM for insomnia should incorporate measures to assess factors that potentially moderate not only the relationship between attentional bias and insomnia, but also any therapeutic effect. To that end, an ongoing randomized controlled trial of $\mathrm{ABM}$ for insomnia is concurrently examining the role of sleeprelated worry and sleep-associated monitoring in the therapeutic potential of ABM (Akram et al., 2018a,b). One can postulate that further steps in this line of enquiry would be to (1) determine the mechanism of action of ABM, if successful even in a subset of patients; and (2) identify moderators of response to ABM. Price et al. (2016) have highlighted the inter-relatedness of moderators and mediators in the therapeutic potential of ABM in relation to anxiety. In other words, is the mechanism of anxiety symptom reduction following ABM due to the successful reduction of attention bias (mediator), and is that successful reduction contingent on particular moderating factors? The same questions can be posed in the insomnia arena. It is possible that potential moderators of response to $\mathrm{ABM}$ may overlap with those that predict the presence of attention bias, but it is also possible that there may be distinct moderating factors to consider. For example, as suggested in relation to anxiety (Price et al., 2016), potential moderators of therapeutic response to $\mathrm{ABM}$ may be strength of attention bias (i.e., positive responders to ABM may be those exhibiting high attention bias); age; $\mathrm{ABM}$ training setting (lab vs. home); and clinician assessed outcomes. These are worthy considerations for future trials examining efficacy of $\mathrm{ABM}$ in insomnia.

A recent review tentatively supports the notion of a sleeprelated attentional bias in insomnia based on six out of nine studies which confirm group differences in relation to attentional allocation to sleep-related stimuli (Harris et al., 2015). However, the number of studies conducted to date still remains limited. Whilst it is possible that a publication bias exists precluding studies demonstrating null effects, to the best of our knowledge, only a further seven studies have been conducted (Woods et al., 2013; Beattie et al., 2017; Akram et al., 2018a,b,c; Koranyi et al., 2018; Zheng et al., 2018; Zhou et al., 2018), of which four provide additional support (Beattie et al., 2017; Akram et al., 2018a,b,c; Koranyi et al., 2018). Therefore, we suggest that further research is required to clarify the presence of a sleep-related attentional bias in insomnia. Additionally, research should pursue the role of potential factors moderating the sleep-related attentional bias/insomnia relationship. In turn, this may allow a particular sub-set of insomnia patients to benefit therapeutically from $A B M$ through appropriate screening.

\section{AUTHOR CONTRIBUTIONS}

All authors listed have made a substantial, direct and intellectual contribution to the work, and approved it for publication.

\section{ACKNOWLEDGMENTS}

The authors would like to thank the reviewers, for providing their expert opinion and insight. 


\section{REFERENCES}

Akram, U., Beattie, L., Ypsilanti, A., Reidy, J., Robson, A., Chapman, A. J., et al. (2018a). Sleep-related attentional bias for tired faces in insomnia: evidence from a dot-probe paradigm. Behav. Res. Ther. 103, 18-23. doi: 10.1016/j.brat.2018.01.007

Akram, U., Ellis, J. G., Myachykov, A., and Barclay, N. (2016). L. Misperception of tiredness in young adults with Insomnia. J. Sleep. Res. 25, 466-474. doi: $10.1111 /$ jsr.12395

Akram, U., Milkins, B., Ypsilanti, A., Reidy, J., Lazuras, L., Stevenson, J., et al. (2018b). The therapeutic potential of attentional bias modification training for insomnia: study protocol for a randomised controlled trial. Trials 19:567 doi: 10.1186/s13063-018-2937-4

Akram, U., Robson, A., and Ypsilanti, A. (2018c). Sleep-related attentional bias for faces depicting tiredness in insomnia: evidence from an eye-tracking study. J. Clin. Sleep Med. 14, 959-965. doi: 10.5664/jcsm.7160

Armstrong, T., and Olatunji, B. O. (2012). Eye tracking of attention in the affective disorders: a meta-analytic review and synthesis. Clin. Psych. Rev. 32, 704-723. doi: 10.1016/j.cpr.2012.09.004

Baglioni, C., Lombardo, C., Bux, E., Hansen, S., Salveta, C., Biello, S., et al. (2010). Psychophysiological reactivity to sleep-related emotional stimuli in primary insomnia. Behav. Res. Ther. 48, 467-475. doi: 10.1016/j.brat.2010.01.008

Baglioni, C., Spiegelhalder, K., Regen, W., Feige, B., Nissen, C., Lombardo, C., et al. (2014). Insomnia disorder is associated with increased amygdala reactivity to insomnia-related stimuli. Sleep 37, 1907-1917. doi: 10.5665/sleep.4240

Barclay, N. L., Eley, T. C., Mill, J., Wong, C. C., Zavos, H. M., Archer, S. N., et al. (2011). Sleep quality and diurnal preference in a sample of young adults: associations with 5HTTLPR, PER3, and CLOCK 3111. Am. J. Med. Genet. B 156B, 681-690. doi: 10.1002/ajmg.b.31210

Barclay, N. L., and Ellis, J. G. (2013). Sleep-related attentional bias in poor verses good sleepers is independent of affective valence. J. Sleep Res. 22, 414-421. doi: $10.1111 /$ jsr.12035

Barclay, N. L., and Gregory, A. M. (2010). The presence of a perseverative iterative style in poor vs. good sleepers. J. Behav. Ther. Exp. Psychiatry 41, 18-23. doi: 10.1016/j.jbtep.2009.08.003

Beattie, L., Bindemann, M., Kyle, S. D., and Biello, S. M. (2017). Attention to beds in natural scenes by observers with insomnia symptoms. Behav. Res. Ther. 92, 51-56. doi: 10.1016/j.brat.2017.02.001

Beevers, C. G., Wells, T. T., Ellis, A. J., and McGeary, J. E. (2009). Association of the serotonin transporter gene promoter region (5-HTTLPR) polymorphism with biased attention for emotional stimuli. J. Ab. Psych. 118:670. doi: 10.1037/a0016198

Brummett, B. H., Krystal, A. D., Ashley-Koch, A., Kuhn, C. M., Züchner, S., Siegler, I. C., et al. (2007). Sleep quality varies as a function of 5-HTTLPR genotype and stress. Psychosom. Med. 69, 621-624. doi: 10.1097/PSY.0b013e31814b8de6

Carney, C. E., Harris, A. L., Moss, T. G., and Edinger, J. D. (2010). Distinguishing rumination from worry in clinical insomnia. Behav. Res. Ther. 48, 540-546. doi: 10.1016/j.brat.2010.03.004

Deuschle, M., Schredl, M., Schilling, C., Wüst, S., Frank, J., Witt, S. H., et al. (2010). Association between a serotonin transporter length polymorphism and primary insomnia. Sleep 33:343. doi: 10.1093/sleep/33.3.343

Espie, C. A. (2002). Insomnia: conceptual issues in the development, persistence, and treatment of sleep disorder in adults. Annu. Rev. Psychol. 53, 215-243. doi: 10.1146/annurev.psych.53.100901.135243

Espie, C. A., Broomfield, N. M., Macmahon, K. A. M., Macphee, L. M., and Taylor, L. M. (2006). The attention-inattention effort pathway in the development of psychophysiologic insomnia: a theoretical review. Sleep Med. Rev. 10, 215-245. doi: 10.1016/j.smrv.2006.03.002

Fortier-Brochu, É., Beaulieu-Bonneau, S., Ivers, H., and Morin, C. M. (2012). Insomnia and daytime cognitive performance: a meta-analysis. Sleep Med. Rev. 16, 83-94. doi: 10.1016/j.smrv.2011.03.008

Fox, E., Ridgewell, A., and Ashwin, C. (2009). Looking on the bright side: biased attention and the human serotonin transporter gene. Pro. Royal. Soc. Bio. Sci. 276, 1747-1751. doi: 10.1098/rspb.2008.1788

Goldman-Mellor, S., Caspi, A., Gregory, A. M., Harrington, H., Poulton, R., and Moffitt, T. E. (2015). Is insomnia associated with deficits in neuropsychological functioning? Evidence from a population-based study. Sleep 38, 623-631. doi: $10.5665 /$ sleep. 4584
Gustavson, D. E., du Pont, A., Whisman, M. A., and Miyake, A. (2018). Evidence for transdiagnostic repetitive negative thinking and its association with rumination, worry, and depression and anxiety symptoms: a commonality analysis. Collabra Psych. 4:13. doi: 10.1525/collabra.128

Hariri, A. R., Mattay, V. S., Tessitore, A., Kolachana, B., Fera, F., Goldman, D., et al. (2002). Serotonin transporter genetic variation and the response of the human amygdala. Science 297, 400-403. doi: 10.1126/science.1071829

Harris, K., Spiegelhalder, K., Espie, C. A., MacMahon, K. M. A., Woods, H. C., and Kyle, S. (2015). Sleep-related attentional bias in insomnia: a state-of-the-science review. Clin. Psychol. Rev. 42, 16-27. doi: 10.1016/j.cpr.2015.08.001

Harvey, A. G. (2002). A cognitive model of insomnia. Behav. Res. Ther. 40, 869-893. doi: 10.1016/S0005-7967(01)00061-4

Harvey, A. G., and Greenall, E. (2003). Catastrophic worry in primary insomnia. J. Behav. Ther. Exp. Psych. 34, 11-23. doi: 10.1016/S0005-7916(03)00003-X

Heinz, A., Smolka, M. N., Braus, D. F., Wrase, J., Beck, A., Flor, H., et al. (2007). Serotonin transporter genotype (5-HTTLPR): effects of neutral and undefined conditions on amygdala activation. Biol. Psychiatry 61, 1011-1014. doi: 10.1016/j.biopsych.2006.08.019

Hirsch, C. R., and Mathews, A. (2012). A cognitive model of pathological worry. Behav. Res. Ther. 50, 636-646. doi: 10.1016/j.brat.2012.06.007

Huang, C., Li, J., Lu, L., Ren, X., Li, Y., Huang, Q., et al. (2014). Interaction between serotonin transporter gene-linked polymorphic region (5-HTTLPR) and jobrelated stress in insomnia: a cross-sectional study in Sichuan, China. Sleep Med. 15, 1269-1275. doi: 10.1016/j.sleep.2014.01.023

Jansson, M., and Linton, S. J. (2006). The development of insomnia within the first year: a focus on worry. B. J. Health Psych. 11, 501-511. doi: $10.1348 / 135910705 X 57412$

Jansson-Fröjmark, M., Bermås, M., and Kjellén, A. (2012). Attentional bias in insomnia: the dot-probe task with pictorial stimuli depicting daytime fatigue/malaise. Cog. Ther. Res. 37, 534-546. doi: 10.1007/s10608-012-9486-z

Kendall, P. C., and Ingram, R. (1987). "The future for cognitive assessment of anxiety: Let's get specific," in Anxiety and Stress Disorders: Cognitive-Behavioral Assessment and Treatment, eds L. Michelson and L. M. Ascher (New York, NY: Guilford Press), 89-104.

Koranyi, N., Meinhard, M., Bublak, P., Witte, O. W., and Rupprecht, S. (2018). Automatic affective responses towards the bed in patients with primary insomnia: evidence for a negativity bias. J. Sleep Res. 27, 215-219. doi: $10.1111 /$ jsr.12591

Lancee, J., Eisma, M. C., van Zanten, K. B., and Topper, M. (2017a). When thinking impairs sleep: trait, daytime and nighttime repetitive thinking in insomnia. Behav. Sleep Med. 15, 53-69. doi: 10.1080/15402002.2015.1083022

Lancee, J., Yasiney, S. L., Brendel, R. S., Boffo, M., Clarke, P. J. F., and Salemink, E. (2017b). Attentional bias modification training for insomnia: A double-blind placebo controlled randomized trial. PLOS ONE. 12:e0174531. doi: 10.1371/journal.pone. 0174531

Lundh, L. G., Froding, A., Gyllenhammar, L., Broman, J. E., and Hetta, J. (1997). Cognitive bias and memory performance in patients with persistent insomnia. Cog Behav. Ther. 26, 27-35

MacLeod, C., and Grafton, B. (2016). Anxiety-linked attentional bias and its modification: illustrating the importance of distinguishing processes and procedures in experimental psychopathology research. Behav. Res. Ther. 86, 68-86. doi: 10.1016/j.brat.2016.07.005

MacLeod, C., Rutherford, E., Campbell, L., Ebsworthy, G., and Holker, L. (2002). Selective attention and emotional vulnerability: assessing the causal basis of their association through the experimental manipulation of attentional bias. J. Ab. Psych. 111:107. doi: 10.1037/0021-843X.111.1.107

Marks, K. R., Roberts, W., Stoops, W. W., Pike, E., Fillmore, M. T., and Rush, C. R. (2014). Fixation time is a sensitive measure of cocaine cue attentional bias. Addiction 109, 1501-1508. doi: 10.1111/add.12635

Milkins, B., Notebaert, L., MacLeod, C., and Clarke, P. J. (2016). The potential benefits of targeted attentional bias modification on cognitive arousal and sleep quality in worry-related sleep disturbance. Clin. Psych. Sci. 4, 1015-1027. doi: $10.1177 / 2167702615626898$

Munafò, M. R., Brown, S. M., and Hariri, A. R. (2008). Serotonin transporter (5HTTLPR) genotype and amygdala activation: a meta-analysis. Bio. Psychiatry 63, 852-857. doi: 10.1016/j.biopsych.2007.08.016

O'Kearney, R., and Pech, M. (2014). General and sleep-specific worry in insomnia. Sleep Bio. Rhyt. 12, 212-215. doi: 10.1111/sbr.12054 
Orff, H. J., Drummond, S. P., Nowakowski, S., and Perlis, M. L. (2007). Discrepancy between subjective symptomatology and objective neuropsychological performance in insomnia. Sleep 30, 1205-1211. doi: 10.1093/sleep/30.9.1205

Perez-Edgar, K., Bar-Haim, Y., McDermott, J. M., Gorodetsky, E., Hodgkinson, C. A., Goldman, D., et al. (2010). Variations in the serotonin-transporter gene are associated with attention bias patterns to positive and negative emotion faces. Bio. Psychol. 83, 269-271. doi: 10.1016/j.biopsycho.2009. 08.009

Pezawas, L., Meyer-Lindenberg, A., Drabant, E. M., Verchinski, B. A., Munoz, K. E., Kolachana, B. S., et al. (2005). 5-HTTLPR polymorphism impacts human cingulate-amygdala interactions: a genetic susceptibility mechanism for depression. Nat. Neurosci. 8, 828-834. doi: 10.1038/nn1463

Price, R. B., Wallace, M., Kuckertz, J. M., Amir, N., Graur, S., Cummings, L., et al. (2016). Pooled patient-level meta-analysis of children and adults completing a computer-based anxiety intervention targeting attentional bias. Clin. Psychol. Rev. 50, 37-49. doi: 10.1016/j.cpr.2016.09.009

Ressler, K. J., and Nemeroff, C. B. (2000). Role of serotonergic and noradrenergic systems in the pathophysiology of depression and anxiety disorders. Dep. Anx. 12, 2-19. doi: 10.1002/1520-6394(2000)12:1+ <2::AID-DA2>3.0.CO;2-4

Spiegelhalder, K., Espie, C., Nissen, C., and Riemann, D. (2008). Sleeprelated attentional bias in patients with primary insomnia compared with sleep experts and healthy controls. J. Sleep Res. 17, 191-196. doi: 10.1111/j.1365-2869.2008.00641.x

Spiegelhalder, K., Kyle, S. D., Feige, B., Prem, M., Nissen, C., Espie, C. A., et al. (2010). The impact of sleep-related attentional bias on polysomnographically measured sleep in primary insomnia. Sleep. 33, 107-112. doi: 10.1093/sleep/33.1.107

Tang, N. K., and Harvey, A. G. (2006). Altering misperception of sleep in insomnia: behavioral experiment versus verbal feedback. J. Consult. Clin. Psychol. 74:767. doi: 10.1037/0022-006X.74.4.767

van Dalfsen, J. H., and Markus, C. R. (2015). Interaction between 5-HTTLPR genotype and cognitive stress vulnerability on sleep quality: effects of subchronic tryptophan administration. Int. J. Neuropsychopharmacol. 18:pyu057. doi: 10.1093/ijnp/pyu057
Van Den Berg, J. F., Van Rooij, F. J., Vos, H., Tulen, J. H., Hofman, A., Miedema, H. M., et al. (2008). Disagreement between subjective and actigraphic measures of sleep duration in a population-based study of elderly persons. J. Sleep Res. 17, 295-302. doi: 10.1111/j.1365-2869.2008.00638.x

Watts, F. N., Coyle, K., and East, M. P. (1994). The contribution of worry to insomnia. B. J. Clin. Psychol. 33, 211-220. doi: 10.1111/j.2044-8260.1994.tb01115.x

Weise, S., Ong, J., Tesler, N. A., Kim, S., and Roth, W. T. (2013). Worried sleep: 24-h monitoring in high and low worriers. Biol. Psychol. 94, 61-70. doi: $10.1016 /$ j.biopsycho.2013.04.009

Wicklow, A., and Espie, C. A. (2000). Intrusive thoughts and their relationship to actigraphic measurement of sleep: towards a cognitive model of insomnia. Behav. Res. Ther. 38, 679-693. doi: 10.1016/S0005-7967(99)00136-9

Woods, H. C., Scheepers, C., Ross, K. A., Espie, C. A., and Biello, S. M. (2013). What are you looking at? Moving toward an attentional timeline in insomnia: A novel semantic eye tracking study. Sleep 36, 1491-1499. doi: 10.5665/sleep.3042

Zheng, S., Feng, J., Lin, R., Yan, Y., Zhang, R., Huang, H., et al. (2018). The impact of negative mood state on sleep-related attentional bias in insomnia. J. Sleep Res. 23:e12748. doi: 10.1111/jsr.12748

Zhou, N., Zhao, C., Yang, T., Du, S., Yu, M., and Shen, H. (2018). Attentional bias towards sleep-related stimuli in insomnia disorder: a behavioural and ERP study. J. Sleep Res. 27:e12652. doi: 10.1111/jsr.12652

Conflict of Interest Statement: The authors declare that the research was conducted in the absence of any commercial or financial relationships that could be construed as a potential conflict of interest.

Copyright (๑) 2018 Akram, Barclay and Milkins. This is an open-access article distributed under the terms of the Creative Commons Attribution License (CC BY). The use, distribution or reproduction in other forums is permitted, provided the original author(s) and the copyright owner(s) are credited and that the original publication in this journal is cited, in accordance with accepted academic practice. No use, distribution or reproduction is permitted which does not comply with these terms. 